\title{
Plantas americanas con uso en medicina en el Diccionario de Terreros y Pando
}

\section{Bertha M. Gutiérrez Rodilla Universidad de Salamanca}

\section{INTRODUCCIÓN}

Es un hecho bien conocido que fueron muchas -y en campos muy diversos- las repercusiones que tuvo para España el encuentro con el continente americano y su posterior colonización. Incluso una vez concluida la conquista, las expediciones que se llevaron a cabo en los siglos siguientes, promovidas con distintos fines, continuaron teniendo fecundas consecuencias. No solamente se importaron de América especias y perfumes, alimentos, tintes, drogas de todo tipo o nuevos materiales para los que nuestro país sirvió de puerta natural de entrada hacia el resto de Europa, sino que con el descubrimiento del Nuevo Continente se completó la organización integral del mundo ${ }^{1}$. Entre dichas expediciones ${ }^{2}$ no son las menos importantes las que se realizaron con fines científicos, relacionadas fundamentalmente con la astronomía y la botánica ${ }^{3}$, en los siglos XVIII y XIX, expediciones y repercusiones que han sido estudiadas, más o menos detenidamente, y con mayor o menor acierto.

La impronta que dejaron todos esos viajes en distintos ámbitos, incluido el de las costumbres cotidianas, es innegable. Por esta razón, es lógico que no escapara de ella tampoco la lexicografía del momento; aunque, curiosamente, a pesar del papel que ésta desempeña como notaria de la recepción de palabras en una lengua y, por ello, notaria de la vida al fin

1 Vid. F. Giral González (1980), p. 46.

2 Vid., por ejemplo, la recopilación que hace A.Guirao de Vierna (1988).

3 El interés por la botánica se puso de manifiesto desde el comienzo de la aventura americana y se mantuvo durante todo el tiempo que ésta duró. El contraste existente entre la atención que le concedieron los españoles y la negligencia de que parece dieron pruebas los primeros colonos del norte de ese mismo continente ha sido ya señalado (vid. A. Tovar, 1970, p. 46). 
y al cabo, no suelen figurar los diccionarios entre las fuentes bibliográficas consultadas y manejadas en la realización del tipo de estudios que antes hemos mencionado.

Pocas veces la lexicografía ha actuado de una forma tan práctica como en las primeras épocas de la conquista: el descubrimiento de un continente nuevo lleno de realidades diferentes a las que se está acostumbrado y la intención de explicar todo lo novedoso que aparece en él, conduce a la necesidad de definir, que es el fundamento de la lexicografía. Por esta razón, a partir de 1492 se escribieron auténticos diccionarios -aunque sin la estructura formal que les damos en la actualidad- llenos de definiciones de esas nuevas realidades, cuyos autores derrochan montañas de imaginación, tanto en las explicaciones que de ellas daban, como en la introducción o formación de neologismos para nombrarlas ${ }^{4}$.

Pero esa capacidad receptora no se desvanece en los siglos de la Ilustración en los que se ponen los fundamentos de la lexicografía actual. Uno de los dos grandes diccionarios dieciochescos - pasados ya más de doscientos años desde la conquista-, el de Esteban de Terreros y Pando (1786-1793), muestra una actitud muy receptiva hacia la realidad de la flora americana y precisamente con respecto a aquellas plantas usadas en la medicina. El hecho se explica por ser la materia médica un dominio donde tuvo lugar una de las más importantes asimilaciones de las cosas nuevas traídas de allís; pero además porque su autor, jesuita, tenía una magnífica información de primera mano de sus propios compañeros de la Compañía, excelentes conocedores -y descriptores- de la realidad americana ${ }^{6}$. Se trata de una obra que se publica, no obstante, antes de que se realizaran las grandes expediciones de mediados del XVIII y principios del XIX, debidas al auge de la Botánica en España, propiciado por un nuevo interés existente en el conocimiento de las colonias de ultramar y,

4 Por ejemplo, en este sentido, Alonso de Molina en 1571 advertía a sus lectores del hechode que no se privaría de crear neologismos tanto en nahuatl como en español, para dar cuenta denociones que no existieran en la lengua objeto $(\mathrm{Cl}$. Boisson, $\mathrm{P}$. Kirtchuk y H. Bejoint, 1991, pp. 291-292).

5 Si bien la actitud de los médicos europeos en este punto fue, como es sabido, encontrada: unos consideraron la medicina americana como algo fantástico y primitivo, asentada en la ignorancia y la superstición y otros, en cambio, la sobrevaloraron; por eso, muchos de los remedios aprovechables que llegaron de América tuvieron que irse abriendo paso muy poco a poco en Europa.

6 Vid. J. Gutiérrez Cuadrado y J. A. Pascual Rodríguez (1996). 
particularmente, de su flora 7 .

La Ilustración española suele considerarse como una especie de resultado atenuado de las "luces" francesas, de forma que Feijóo es un enciclopedista mitigado, Luzán, un mero reflejo del clasicismo francés e italiano y la lexicografía académica se origina por el impulso que los Borbones dieron a nuestra lengua, a imitación del trabajo que desarrollaba la Academia francesa. La importancia de las obras españolas se mide, pues, aplicando el rasero de lo que acaecía en los círculos europeos, sin contar ni siquiera con la posibilidad de que lo que se hacía exclusivamente en España pudiera suponer alguna ventaja frente al modo de trabajar de los demás países. En este sentido, Terreros ha sido interpretado como un diletante cuyos esfuerzos superaron a los logros obtenidos en su trabajo; este desfase se debería a que su intención procede de la modernidad, pero la información de que dispone no pertenece precisamente a la vanguardia europea. De este modo, ha pasado con menos gloria de la que debiera una obra que resulta única entre todas las europeas de su tiempo: prácticamente el primer diccionario concebido, no sólo como de lengua, sino científico a la vez. Sarrailh colocó al Padre Terreros como un subproducto de los diccionarios científicos europeos (vid. J. Sarrailh, 1957, p. 457), dado que -y sus apoyos son tan dudosos como el Abate Alea y Sempere y Guarinos- en el siglo XVIII el vocabulario científico no era familiar a los españoles, por estar desprovistos éstos de diccionarios de ciencias. No era ésta, sin embargo, una condición exclusiva de España, sino del conjunto de Europa, con lo que la obra de Terreros cambia absolutamente de valor, al ser pionera en el ámbito de la lexicografía científica. Y cabe añadir, además, que, en algunos campos específicos, como el de la medicina por ejemplo, fue también en la mente de un español, y en este mismo siglo XVIII, donde se concibió la idea de escribir el primer diccionario médico con características modernas en Europa ${ }^{8}$.

\section{LA MEDICINA Y LAS PLANTAS MEDICINALES AMERICANAS EN EL DICCIONARIO DE TERREROS}

Recoge el jesuita vasco en su diccionario gran cantidad de tecnicis$\operatorname{mos}^{9}$ que recopila en sus labores de traducción y, desde luego, en sus

7 Vid. I. del Campo (1993), p. 11.

8 A pesar de que ese honor se lo haya concedido la historia al británico Robert James (vid. B. M. Gutiérrez Rodilla, en prensa). 
abuntísimas lecturas. En el otro gran repertorio español del Setecientos -el Diccionario de Autoridades-, por sus propios planteamientos previos, los tecnicismos no tenían cabida; y si, no obstante, se hace eco de muchos ${ }^{10}$, no resultan estos comparables a la gran cantidad que de ellos incluye Terreros. Este no sólo se limita a traducir sino que, cuando se encuentra un término -fundamentalmente en francés- que no tiene correspondencia en español, procede a la creación de un equivalente, adaptando generalmente el original a la estructura fonológica del español; con ello su obra se convierte así, para una buena parte de los términos que se recogen en ella, en la primera fuente escrita que existe en nuestra lengua.

La representación de la medicina en su diccionario, aunque sea más racional y completa que en el de Autoridades, no alcanza tampoco unos niveles excelentes, desde el punto de vista de la homogeneidad: unas áreas tienen mucha mayor presencia que otras y resulta desigual el trato que se da dentro de cada parcela a los diferentes términos que se incluyen en ella. Es impensable -y hasta hubiera resultado anacrónico- que Terreros pudiera realizar su diccionario habiendo sopesado previamente qué términos iban a entrar y cuáles no, en razón de su representatividad, estableciendo porcentajes o cuotas previas... Para la medicina americana los planteamientos no iban a ser diferentes: son muchas las plantas medicinales incluidas, pero más bien escasas las enfermedades o nombres de enfermedades específicas "americanas", así como los remedios preservativos o curativos que no tengan que ver con las plantas, como, por ejemplo, el empleado contra el cólera que, sin duda, llamó su atención

En Indias le llaman Maudechin, y el remedio que le aplican, es quemarle las plantas de los pies á quien la tiene, y no dejarle bebér.

o el chimóo, un extraño "dentífrico" que es

un compuesto del espiritu del tabaco, y de un barro, que sacan de una laguna, que está cerca de la Ciudád de Mérida en la América, [...] este compuesto usan todos los vecínos de aquella Ciudád para frotár la dentadura, y librárla del daño que le causa una sierra nevada, que domina la Ciudad: el

9 Sobre este aspecto en el diccionario de Terreros vid., por ejemplo, la presentación que hace M. Alvar Ezquerra (1987, 1993), P. Álvarez de Miranda (1992) o B. M. Gutiérrez Rodilla (1996).

10 Vid. B. M. Gutiérrez Rodilla (1993). 
modo de valerse de él es traherle en unas cajitas de plata, y sacando con una cucharíta un poco, estriegan con ello dientes, y muelas; pero fuera de no librarse del todo con esta medicina, causa sumo asco la saliva que arrojan.

No estaba en el ánimo de Terreros hacer un diccionario de americanismos que recogiera los variados términos que podían emplearse más allá de los mares para las realidades que aquí ya se conocían antes del descubrimiento, por lo que en las equivalencias que se proporcionan en el último tomo del diccionario no hay ninguna lengua no europea. Por ello, la inclusión tan abundante de nombres "americanos" de plantas responde a que se trataba de referentes nuevos que necesitaban de sus respectivas designaciones, y que, por lo tanto, los que hasta ese momento eran "simples" desconocidos podían llegar a convertirse en corrientes, cotidianos, para los españoles ${ }^{11}$. Los pocos nombres de enfermedades que introdujo seguramente respondían a la misma consideración: pudo pensar que se encontraba ante situaciones nuevas no conocidas en España antes del descubrimiento, por más que tratara de facilitar su comprensión relacionándolas con conceptos comunes en nuestro país: así el cocoliste es un "tabardillo de tripas", el coto una "especie de lupia de América", el courap un "nombre que dán los Indios á una especie de herpes" o el conocido zurumpi, que es un "vehementísimo dolor de ojos".

Como hemos avanzado, será el repertorio de Terreros la primera obra lexicográfica - sino la única- que recoja muchas de las plantas que llegaron a España y a Europa procedentes de los otros tres continentes conocidos y bien explorados hasta aquel momento: Asia, Africa y América12. Las incluídas en el diccionario del jesuíta con aplicación en medicina son, en términos globales, 24713 , lo que significa el 5,06\% del total de términos relacionados con la medicina que incluye este diccionario (4.873). De entre ellas, las que proceden de América suman 162, es

11 Al fin y al cabo la inclusión de todas aquellas designaciones en su diccionario tuvo lugar en el preciso momento en que iba a producirse el despegue de la botánica en nuestro país y cuando se estaban librando las batallas principales por la "modernización" de la química y la farmacia españolas (vid., por ejemplo, A. González Bueno y F. J. Puerto Sarmiento,1988, pp. 133-139).

12 El quinto continente se empezó a explorar a comienzos del XVII, pero su conocimiento no se dió por completado hasta finales del XVIII.

${ }^{13} \mathrm{El}$ dato se refiere a entradas diferentes, pero hay que contar con que en ocasiones existen sinónimos -aunque no sea muy frecuente- por lo que dos o más entradas pueden referirse a una misma planta. 
decir, dos terceras partes. Siguiendo una división, que nos parece muy oportuna, establecida por Pardo Tomás y López Terrada en su trabajo sobre plantas americanas (J. Pardo Tomás y M. L. López Terrada, 1993, pp. 199200), nosotros sólo hemos contado para el nuestro con las calificadas por ellos como los "nuevos remedios viejos" que eran nuevas pero que, por su similitud aparente o por tener un uso común con el de sus parientes empleados en Europa, fueron asimiladas con relativa facilidad, y con las plantas calificadas como "nuevas medicinas" hasta entonces completamente desconocidas; es decir, los grupos dos y tres de su clasificación ${ }^{14}$.

Esteban de Terreros, tan metódico para otras cosas, es extraordinariamente asistemático a la hora de indicar la procedencia geográfica de las plantas que incluye en su obra, por lo que no siempre es fácil saber exactamente su origen. Hemos contabilizado entre las plantas "americanas", no sólo aquellas en las que se indica explícitamente que lo son, sino también otras de las que se apunta que proceden de ambas Indias (orientales y occidentales), algunas de las que se informa que vienen de Indias, sin mayor precisión, e incluso algunas de las que no se dice nada pero de las que conocemos, por otras fuentes, su origen americano. Todo ello nos da el siguiente resultado para dicha procedencia: Indias (30), América (30), Brasil (28), Nueva España (10), Indias Occidentales (8), Perú (7), California (4), Méjico (3), Mechoacán (3), La Florida (2), Jamaica (2), Antillas (2), Campeche (2), Santa Cruz (2), Ambas Indias (Orientales y Occidentales) (2), Santa Marta (1), Quito (1), Popayán (1), Los Mojos (1), Nuevo Méjico (1), países cálidos (1), Maldivas (1), Cumaná (1), Guadalupe (1), Cayena (1), Santa Fé (1), Orinoco (1), Chile (1) y 14 sin especificar ${ }^{15}$.

Los nombres de las plantas que proporciona el jesuíta son también extremadamente heterogéneos: junto a los que podríamos considerar como españoles, están los recogidos en las diferentes lenguas vernáculas y aun algunos adaptados al español del latín o del francés, todo ello según las fuentes de donde los tomara. Y esto sin contar con la previa confusión terminológica que sufrieron los autores en los que se documenta Terreros, quienes generalmente utilizaban tanto el nombre técnico que se podía dar

${ }^{14} \mathrm{El}$ grupo uno es el formado por plantas que existían desde siempre en el viejo mundo y que se encontraron también y reconocieron al llegar al nuevo.

15 Somos conscientes de que Nueva España es el nombre dado por H. Cortés a Méjico y de que Michoacán, Campeche y Nuevo Méjico también se encuentran en Méjico, pero hemos respetado las diferencias que establece Terreros en las procedencias. 
a una planta, como las denominaciones vulgares, o bien las latinas, aún más confusas, lo que origina denominaciones de toda procedencia: técnica, vulgar, española, latina, americana, francesa...16. Razones todas que, en conjunto, hacen que, en muchos casos, sea prácticamente imposible saber a qué planta en concreto se refiere, o si una misma planta responde a dos o más nombres diferentes.

\section{FUENTES UTILIZADAS}

Una de las diferencias formales entre el diccionario de Terreros y el de la Academia es la presencia de "autoridades" que avalan los términos que se incluyen; la gran cantidad de ellas que aparecen en el repertorio académico le ha proporcionado precisamente el sobrenombre por el que se le conoce, en tanto que la consignación de las fuentes manejadas por el jesuíta es bastante inferior ${ }^{17}$.

En el caso de las plantas con uso en medicina venidas de América, Terreros nos informa en 46 ocasiones $(28,39 \%$ del total de plantas) de las fuentes tan diferentes que utilizó. Estas son, desde luego, muy variadas: obras históricas, literarias, repertorios lexicográficos o libros de viajes. Así, encontramos en primer lugar por número de veces que se cita, el Espectáculo de la Naturaleza... de Noel Pluche, que el propio Terreros había traducido desde el francés (1753-1755) y que es también la obra no médica más citada para ilustrar otros términos relacionados con la medicina incluídos en este diccionario ${ }^{18}$; obras literarias como Pastores de

16 Esto sin tomar en consideración las distintas realizaciones que se pueden dar según autores o zonas a una misma denominación, como ocurre, por ejemplo, con saxifraga, también conocida como salsafras, salsifras, salsifrajia, calafraga, sasafras, saxifras, saxifragia, etc.

17 Frente al $70 \%$ de las voces médicas que, en el repertorio académico se acompañan de 'autoridad', algo menos del $16 \%$ de las de Terreros lo hacen. Vid., para las fuentes que consigna Terreros para los términos médicos, B. M. Gutiérrez Rodilla (1996) y, para las de Autoridades, B. M. Gutiérrez Rodilla (1994-1995).

18 Que el abate Pluche se dejara llevar en algunas ocasiones por "extrañas" ideas hasta el punto de dar explicaciones demasiado relacionadas con Dios -y hasta con los ángeles- para algunos fenómenos de la naturaleza, no constituye materia suficiente, en nuestra opinión, para descalificar toda su obra como hace J. Sarrailh (1957, pp. 456458.) Este autor la valora aplicando presupuestos del siglo XX a situaciones del XVII y XVIII; la critica duramente y, en cierto modo, se burla de la aceptación que tuvo entre los españoles; la compara con la de Buffon, al que alaba -a nuestro juicio- desmesuradamente, si tenemos en cuenta que varios aspectos de la obra de éste último, como ocurre por definición siempre que de ciencia se trata, en un momento determinado estuvieron igual de desfasados que los de la de Pluche. 
Belén... (1612) de Félix Lope de Vega o El desdén con el desdén (1654) de A. Moreto y Cabana, e "históricas" como la Historia de California19, el Arancel de rentas y diezmos del año de 1709, la Historia natural y moral de las Indias (1590) del Padre José de Acosta, la Historia de la conquista de Mexico... (1684) de Antonio de Solís, el Orinoco Ilustrado... (1741) del Padre J. Gumilla, los Veintiún libros rituales y Monarquía Indiana (1615) de Juan de Torquemada o la Politica Indiana (1648) de Juan de Solórzano, y repertorios lexicográficos como el Diccionario trilingüe...(1745) de Manuel de Larramendi, el Diccionario nuevo de las lenguas española y francesa...(1705) de F. Sobrino, el propio Diccionario de Autoridades (1726-1739), el Diccionario universal francés y español (1743-1744) de Antonio $\mathrm{M}^{\mathrm{a}}$ de Herrero o el Lexicon medicum Graeco-Latinum ex Hippocrate, et Galeno desumptum (1598) de Bartolomeo Castelli, entre otros.

Da cuenta también de haber usado obras médicas y de farmacia, como las Manifiestas demostraciones de las más seguras y suaves curaciones del Morbo galico (1745) del doctor Francisco Suárez de Rivera, el libro De compositione medicamentorum... (1610) de Gaspar Bauhin -autor que dedicó gran parte de su trabajo a la creación de nombres genéricos para las plantas-, el Petitorio pharmaceutico arreglado a la pharmacopea matritense (1753) y De todas las cosas que se traen de nuestras Indias Occidentales que sirven en Medicina...(1565) de Nicolás Monardes.

Cabría establecer otro apartado con los libros de naturaleza y viajes que manejó, como la Flora española o Historia de las plantas que se crían en España (1762), del médico y botánico José Quer Martínez, las Instituciones botánicas (1700, edición latina) de Joseph P. de Tournefort o la Relación histórica del viage a la America meridional... (1748) de Jorge Juan Santacilia y Antonio Ulloa de la Torre-Giral. Menciona también al famoso botánico Antoine de Jussieu, pero resulta imposible saber a cuál de sus obras se refiere, y hasta al embajador francés en Portugal, Jean Nicot que, por esas raras jugadas del destino de los epónimos, dejó para siempre unido su nombre a un género de solanáceas -Nicotiana- y

19 No resulta fácil decidir de entre todos los libros que hay sobre Califomia y su historia a cuál se refiere, pero pensamos -por datos y pistas encontrados a lo largo del diccionario- que es a alguna de las obras que sobre este tema escribió el jesuita $\mathbf{M}$. Venegas, en concreto, M. Venegas (1757). 
al alcaloide nicotina ${ }^{20}$.

Gracias a la información que proporciona en otros términos médicos no relacionados con la terapéutica botánica, podemos completar de alguna manera el espectro de fuentes utilizadas para asesorarse en este campo de las plantas medicinales, aunque no sólo americanas. Entre dichas fuentes están Andrés Laguna y su traducción castellana con comentarios del Pedacio Dioscorides Anazarbeo, acerca de la materia medicinal...(1555), la Traducción de los libros de Caio Plinio Segundo, de la Historia Natural...(1599) de Jerónimo Gómez de Huerta, la Palestra pharmaceutica chymico-galenica (1706) del farmacéutico Félix Palacios y Bayá, la Pharmacologia seu manuductio...(1696) de Samuel Dale, el Curso Chymico... de Nicolas Lémery, traducido del francés y ampliado por Félix Palacios y Bayá en 1703, las Memorias de la Academia Real de las Ciencias de París, de 1729 a 1732, una a la que llama Historia general de las plantas ${ }^{21}$, el Frumentorum, leguminum, palustrium et aquatilium herbarum... (1566) del médico Rembert Dodoens, la Description de plantes de l'Amerique, avec leurs figures... (1693) del Padre Charles Plumier o la Agricultura general y gobierno de la casa de campo... (1765-1795) de José Antonio Valcárcel. Se sirvió finalmente de cartas y relaciones como, por ejemplo, las Cartas edificantes y curiosas, escritas de las misiones estrangeras, por algunos missioneros de la Compañía de Jesús... (1753-1757) o la Relacion enviada de holanda el año de 1763 en orden á las flores que cultivan los floristas Voorhelm y Zompel. No debemos olvidar las fuentes donde Terreros resulta más innovador dentro de la lexicografía, que son las publicaciones periódicas, tales como la Gaceta de Madrid o la Gaceta de Londres.

Como se puede fácilmente apreciar, las obras consultadas por el autor del diccionario que nos ocupa son variadas y heterogéneas pero, por eso precisamente, bastante completas. La intuición de Terreros $-\mathrm{o}$ su erudición- le llevó a conjugar a la perfección la pervivencia de la tradi-

20 El "gran" embajador de quien tomó el nombre Linneo para el género Nicotiana practicaba, al parecer, el espionaje comercial amparado en la inmunidad diplomática que le proporcionaba su cargo y, de esta forma, enviaba a París productos que "pescaba" en las naves portuguesas o españolas que llegaban a Lisboa (vid. F. Giral González, 1980 , p. 81). Otra versión de los hechos puede verse en J. Baudry (1988), donde se presenta a este personaje como un quasi héroe.

${ }^{21}$ Hay tantas historias de plantas que resulta imposible localizar sólo por este título tan inconcreto ésta que utilizó Terreros, por lo que no podemos proporcionar ni autor, ni fecha. 
ción griega renovada y reelaborada por el humanismo, cuyo mejor ejemplo para este caso es la traducción con comentarios de la Materia Medica de Dioscórides hecha por Andrés Laguna ${ }^{22}$, con la obra De todas las cosas que se traen de nuestras Indias Occidentales que sirven en Medicina...(1565), de Nicolás Monardes, el "profesional médico español al que Europa debe el primer conocimiento efectivo de los 'simples medicinales' indianos" (L. S. Granjel, 1991, p. 34). El manejo de estas obras indicadas, pertenecientes a siglos anteriores al suyo, no le impidió tampoco consultar las más recientes y totalmente cercanas a cuando él llevó a cabo el diccionario, como la Relación... (1748), de Jorge Juan y Antonio Ulloa. Pero sobre todo fue importante que usara la Flora española... (1762) de José Quer Martínez, el hombre cuyo jardín fue tomado como base para la creación en 1755 del Jardín Botánico de Migas Calientes, del que fue nombrado primer profesor ${ }^{23}$, y las Instituciones botánicas (1700, edición latina) de Joseph P. de Tournefort, pues no sería hasta 1785 cuando se empezara a abandonar progresivamente en España a Tournefort en favor de la nomenclatura de Linneo ${ }^{24}$.

CARACTERÍSTICAS, VIRTUDES CURATIVAS DE LAS CITADAS PLANTAS Y MODOS DE USARLAS

El espectro de las virtudes curativas de que gozan estas plantas o las enfermedades para las que se suelen utilizar, es bastante amplio y va, desde la que sirve para quitarse los callos de los pies, pasando por la que hace que desaparezcan las pecas de la cara, hasta la que puede considerarse "remedio universal", por servir prácticamente para todo. Basándonos en estas utilidades que se les atribuye, podemos establecer siete apartados con ellas:

a) el grupo de las que tienen una acción purgante;

b) las que actúan sobre el tubo digestivo favoreciendo las digestiones, fortificando el estómago o ayudando a la expulsión de los gases, y las que son eficaces contra las diarreas por su acción astringente;

22 Vid. L. S. Granjel (1991), p. 32.

23 I. del Campo (1993), p. 18.

24 Recordemos que, aunque el diccionario se publique entre 1786 y 1793, para entoncesTerreros ya está muerto. 
c) las útiles en las "calenturas" o fiebres, fundamentalmente tercianas;

d) las que se han mostrado como eficaces contravenenos;

e) las beneficiosas en los cuadros de vías urinarias caracterizados de mil formas diferentes: ardores de riñones, cólicos nefríticos, mal de piedra, encendimiento de orina...;

f) las empleadas contra el "mal gálico" y otros cuadros venéreos; y

g) por último, un grupo numeroso que se podría etiquetar como "de acción dermatológica", de las que se extraen pomadas, ungüentos, bálsamos, jabones medicinales... útiles en diferentes procesos que afectan a la piel, tales como heridas, úlceras, llagas, empeines, callos, lunares, pecas, sarna o erisipela.

En cuanto al elemento de la planta del que se obtiene el principio activo terapéutico, prácticamente no hay parte de estas plantas de donde no se pueda extraer algo que sirva para curar; lo más frecuente es que proceda de la fruta o fruto de un árbol, seguido en orden de frecuencia, por la raíz, las hojas, la corteza, en el caso de que sea un árbol, o la resina. Avanzando un grado más en la escala de la sofisticación, el remedio puede provenir también de la savia de la planta, del hueso del fruto, del aceite del hueso del fruto, de los granitos que tienen algunos frutos, de la flor de la planta, de la corteza que a veces recubre las raíces o del jugo que pueden tener las espinas que se encuentran en algunos árboles. En el nivel más alto de la escala estaría el licor que se halla entre las dos cortezas del fruto de un árbol llamado marañon, el cual tiene

dos cortezas la una algo parda, y la otra negra; y entre ellas hai un licor muy cáustico, que sirve para los empeines, y para hacer caer los callos de los pies, y abrir fuentes en los brazos sin dolor alguno

o el hacer un jabón medicinal a partir de las cenizas de un árbol, como ocurre con el pacal, e, incluso, utilizar los gusanos que se encuentran en un determinado árbol, como en el caso del cuapátli, que

cria una especie de gusanos velludos, asperos, y encarnados, de dos pulgadas de largos, y de la figura de un cañuto. Los Indios los cuecen en agua hasta que se deshagan, y cojen la grasa, que sube á la superficie, y aplicada despues, apacigua todos los dolores en cualquiera parte del cuerpo; resuelve los humores, fortifica los nervios, y con la terebintina, y jugo del tabaco es buena para las hernias. 
Lo expuesto en este apartado nos lleva a matizar la opinión según la cuál "en realidad, la farmacia indígena americana, en los tiempos de la conquista, no ofrecía contraste con la farmacia europea" 25 , pues su reflejo en la lexicografía de la Ilustración muestra que pudo servir para completar el espectro de "simples" con que contar y de enfermedades a tratar médicamente sin el concurso de otros recursos más intervencionistas.

No es infrecuente encontrar que cada parte de una misma planta tenga funciones diferentes como, por ejemplo, el abaremo-temo, árbol del Brasil cuyas hojas tienen la cualidad de ser astringentes, mientras que la corteza, hecha polvo y cocida, es vulneraria; o el guayacán, cuya corteza -el Palo Santo- es bien conocida por sus buenas cualidades contra las enfermedades venéreas, pero cuya fruta es utilizada como laxante. Algunas plantas, como el avellano de Guadalupe, son tan completas que tienen unas hojas con la virtud de curar úlceras antiguas de forma que,

por la parte mas suave las limpian, y ponen encarnadas, consumiendo la carnosidad, ó parte dañada; y estando en este estado, las acaba de curar la parte inferior, ó aspera de la misma hoja.

En otras ocasiones ocurre que una misma parte de una planta tiene varias funciones diferentes, como la cocción de la raíz del carameis, que sirve tanto contra el asma como contra las calenturas, o la fruta del árbol llamado carcápuli, la cual

modera la diarréa, excita el apetíto, facilíta el parto, aumenta la leche de las que crian; y hecha polvos aclara la vista soplandolos á los ojos,

o la poderosa raíz de acuitce-huariacua, que

apacigua la calentura, y fortifica el corazon, es contraveneno seguro, y resiste á las picaduras ponzoñosas, en particular del Escorpion; fuera de esto templa el ardor de riñones, y el encendimiento de la orina, modera los dolores de pecho, quita los tumores de la garganta, y la quieren hacer pasar por un remedio universal. 
En cuanto al modo de utilización de todas estas plantas, lo normal es que se ingieran tras haber sido reducidas a polvos, o se apliquen directamente sobre el cuerpo en el caso de aceites, resinas, savias, etc. Pero hay también formas más o menos curiosas de hacer uso de ellas como los polvos del apoyomatli, que para que ejerzan su efecto deben ser echados en vino, o los del blaso que habrían de tomarse con jenjibre, o la fruta del aracamiri, a partir de la cuál se hace un "dulce" que es el que ejerce la función curativa. La semilla del cropiot se mezcla con el tabaco, la raíz de la guaparaiba, para que ejerza su acción conta la picadura del Niqui, debe tostarse previamente, y la de capuupeba se toma en infusión. Los renuevos del mizquitzl, se ponen en infusión y el agua que se obtiene se comporta como excelente colirio para las afecciones de los ojos. Hay incluso una planta, cebi-píra, de cuyos beneficios puede uno gozar cuando la utiliza en forma de baño.

El diccionario de Terreros es reflejo de una sociedad en que late todavía con fuerza el pulso de la realidad americana. No nacen las expediciones científicas posteriores de la nada sino que son la continuación de la percepción de aquel mundo, que nuestra lengua - como nuestros barcos- fue la primera en desvelar.

Si Esteban de Terreros introdujo tantas plantas medicinales americanas en su diccionario es porque está reflejando un interés por la realidad propio de los círculos científicos más avanzados, como es el caso del de los jesuítas. Lo difícil es saber hacia dónde nos conducen estas pistas. No se trata de extravagancias o de curiosidades, como podría pensarse viendo los hechos a través de los anteojos que los europeos se habían colocado entonces para observar la realidad, sino de un proceder en el que Terreros fue un auténtico pionero. Y lo fue por su capacidad de reflejar en el plano meramente lexicográfico las ideas comunes a los españoles cultos del momento. Es una actitud que, como historiadores, no nos puede sorprender. Lo sorprendente - y por tanto, lo que habrá que justificar- es que esa labor iniciada por Terreros no tuviera continuación. 


\section{BIBLIOGRAFÍA}

Alvar Ezquerra, M. (1987): "Introducción" a E. DE Terreros, Diccionario castellano con las voces de ciencias y artes y sus correspondientes de las tres lenguas francesa, latina é italiana [ed. facsímile], Madrid, Arco/Libros.

Alvar EzQuerra, M. (1993): "El Diccionario de Terreros", en Lexicografia descriptiva, Barcelona, Biblograf, pp. 249-259.

Álvarez DE MIRANDA, P. (1992): "En torno al Diccionario de Terreros", Bulletin Hispanique, 94, pp. 559-572.

BAUDRY, J. (1988): Jean Nicot, Lyon, La manufacture.

BoIsson, C.L., KIRTCHUCK, P. y BejoINT, H. (1991): "Aux origines de la lexicographie: les premiers dictionnaires monolingues et bilingues", International Journal of Lexicography, 4, pp. 261-315.

CAMPO, I. DEL (1993): Introducción de plantas americanas en España, Madrid, Ministerio de Agricultura, Pesca y Alimentación.

Giral GonZÁlez, F. (1980): Comentarios químico-farmacéuticos a la historia española en América, [discurso de inauguración del curso académico de la Universidad de Salamanca 1980-1981], Salamanca, Ed. Universidad de Salamanca.

González Bueno, A. y Puerto Sarmiento, F. J. (1988): “Ciencia y Farmacia durante la Ilustración", en M. Selles, J. L. Peset y A. Lafuente (comps.), Carlos III y la Ciencia de la Ilustración, Madrid, Alianza Editorial, pp. 127-140.

GRANJEL, L. S. (1991): "Las repercusiones médicas del descubrimiento", en J. Riera (comp.), La medicina en el descubrimiento, Valladolid, Publicaciones Universidad de Valladolid, pp. 29-41.

Guirao De Vierna, A. (1988): "Notas para la clasificación de las expediciones españolas del siglo XVIII a América", en Estudios sobre Historia de la Ciencia y de la Técnica, Valladolid, Junta de Castilla y León, II, pp. 585-595.

GutiérRez CuAdrado, J. y PASCuAl Rodríguez, J. A. (1996): “El trabajo lingüístico de Jolis y la lexicografía novohispana del siglo XVIII", Lexis, 20, pp. 333-371.

GuTIÉrREZ RodiLla, B. M. (1993): "Los términos relacionados con la medicina en el Diccionario de Autoridades", BRAE, 73, pp. 463512.

GuTIÉRRez Rodilla, B. M. (1994-1995): “Construcción y fuentes utilizadas para los términos médicos en el Diccionario de 
Autoridades", Revista de Lexicografia, I, pp. 149-162.

GutiérRez Rodilla, B. M. (1996): "El léxico de la medicina en el diccionario de E. de Terreros y Pando", en A. Alonso González, L. Castro Ramos, B. Gutiérrez Rodilla y J. A. Pascual Rodriguez (eds.), Actas del III Congreso Internacional de Historia de la Lengua Española, [Salamanca, 1993], Madrid, Arco/Libros, II, pp. $1327-1342$.

GutiÉRREZ Rodilla, B. M. (en prensa): "Lo que pudo haber sido y no fue: Francisco Suárez de Rivera y la lexicografía médica moderna", en Actas del I Coloquio sobre la Historia de los lenguajes iberorrománicos de especialidad (s. XVI-XVIII), [Barcelona, 1997].

PARDO TOMÁs, J. y LÓPEZ TERRADA, M. L. (1993): Las primeras noticias sobre plantas americanas en las relaciones de viajes y crónicas de Indias (1493-1553), Valencia, Universitat de València-CSIC.

SARRAILH, J. (1957): La España Ilustrada de la segunda mitad del siglo XVIII, México, Fondo de Cultura Económica.

Terreros y PANDO, E. DE (1786-1793): Diccionario Castellano con las voces de Ciencias y Artes, 4 vols., Madrid, Ibarra. (Utilizamos la ed. facsímile: Madrid, Arco Libros, 1987).

Tovar, A. (1970): Lo medieval en la conquista y otros ensayos americanos, Madrid, Seminarios y Ediciones, S.A.

VAlverde, J.L. y PÉrez Romero, J. A. (1988): Drogas americanas en fuentes de escritores franciscanos y dominicos, Granada, Publicaciones de la Universidad de Granada.

VENEGAS, M. (1757): Noticia de la California, y de su conquista temporal..., 3 vols., Madrid, Viuda de Manuel Fernández, y del Supremo Consejo de la Inquisición. 


\section{APÉNDICE: LISTADO DE PLANTAS}

aavora

acacia verdadera

achanaca

achorou

aloe americano

ambaiba

ana-colupa

anime, ó goma anima

apoyomatli

árbol de toda especie

avellana de la India

ayabutipita

ayubatipita

balsamo de Copahú

bejuquillo

belílli

blaso

caa-ataya

caaetimai

caaobetinga

caapia, ó caa-apia

calafraga

canchelagua, ó canchalagua,

ó canchilagua

cañafistola del Brasíl

carameis

caraschúlli

carin-curini

cásia purgánte

cayán

cebi-píra

coca ó cuca

colcacuahuitl

copal

copaú abaremo-temo

acedera de Indias

aceite de palo

acuitce-huariacua

amatzquitl

ambia-monardi

andira, ó anjelin

aninga

aracamiri

árbol de emborrachar

avellano de Guadalupe

aye

balsamo de Perú

belilla

ben, ó behem

caacíca

caagulínyo

caapeba

cadel-avanacú

calaguála

cañafistola

capuupéba

carapa

carcápuli

cascarilla

cásia-fistola

cayapia

coatli

cohine

contra-yerba

copaljocól

cropiot 
cuapátli

chácos

charapéti

chiantzólli

chimóo

doradilla

espadilla

floripondio

goaconez

guacaténa

guayacán

hermia

holli

ibibiraba

igbucami

jacape

jalapa

jangomas

jenjibre

karata

lilac

macaxocolt

marañon

mateclú

mechoacan

mizquitzl

nhandi cuarango

charameis

chayóte

chibón

chipahuataiztic

drago

estoraque

goma-guta

guaparaiba

guayaco

hiboucouhu

idon-moulli

ipecuacuana

jacua-aganga

jandiroba

janipaba

jua-beba

liquidambar

mamanga

maripenda

mecaxochitl

mezquite

nilica-maram 
nuez medicinal

numularia

pacal

palma christi, o ricino vulgar

palo de María

palo santo

pareira-brava

pimpinichi

queraiba

quina-quina

quinual

saamouna

salsifras o salsifrajia

sangre de drago

saxifras

tabaco

taborucura

tamalapatra

tecomate

tlalamatla

tutuma

vainica, ó vainilla

xalcocotilo

yabacami

yuca

zabida o zabila nuez unguentaria

paico

palo de campeche

palo nefrítico

papaya

pita-haya

pinipinichi

quina

quinoa

salsafras o salsifras

sandalo

sasafras

saxifrajia

tabaco verino

tacamaca

tamarindo

titicana

tlisochitl

vall

xocohuitzles

youli

zarza-parrilla 